Commun. Korean Math. Soc. 20 (2005), No. 1, pp. 169-178

\title{
ON PREECE'S IDENTITY AND OTHER CONTIGUOUS RESULTS
}

\author{
Junesang Choi, Arjun K. Rathie and Bharti Bhojak
}

\begin{abstract}
Five results closely related to the well-known Preece's identity obtained earlier by Choi and Rathie will be derived here by using some known hypergeometric identities. In addition to this, the identities obtained earlier by Choi and Rathie have also been written in a compact form.
\end{abstract}

\section{Introduction and Preliminaries}

From the theory of differential equations, Preece [6] established the following very interesting and useful identity involving product of generalized hypergeometric series:

$$
{ }_{1} F_{1}(\alpha ; 2 \alpha ; x) \cdot{ }_{1} F_{1}(\alpha ; 2 \alpha ;-x)={ }_{1} F_{2}\left(\alpha ; \alpha+\frac{1}{2}, 2 \alpha ; \frac{x^{2}}{4}\right)
$$

Later on, by using the classical Watson's theorem, Bailey [1] derived a generalization of (1.1):

$$
\begin{aligned}
& { }_{1} F_{1}(\alpha ; 2 \alpha ; x) \cdot{ }_{1} F_{1}(\beta ; 2 \beta ;-x) \\
= & { }_{2} F_{3}\left(\frac{1}{2}(\alpha+\beta), \frac{1}{2}(\alpha+\beta+1) ; \alpha+\frac{1}{2}, \beta+\frac{1}{2}, \alpha+\beta ; \frac{x^{2}}{4}\right) .
\end{aligned}
$$

Received March 4, 2004.

2000 Mathematics Subject Classification: Primary 33C65; Secondary 33C60, 33C70 $33 \mathrm{C} 05$.

Key words and phrases: generalized hypergeometric series ${ }_{p} F_{q}$, Dixon's summation formula for ${ }_{3} F_{2}$, Whipple's summation theorem for ${ }_{3} F_{2}$. 
Recently Rathie [8] gave a very short proof of this dentity and obtained the following two results contiguous to (1.1):

$$
\begin{aligned}
& { }_{1} F_{1}(\alpha ; 2 \alpha ; x) \cdot{ }_{1} F_{1}(\alpha ; 2 \alpha+1 ; x) \\
& =e^{x}\left[{ }_{1} F_{2}\left(\alpha ; \alpha+\frac{1}{2}, 2 \alpha ; \frac{x^{2}}{4}\right)\right. \\
& \left.-\frac{x}{2(2 \alpha+1)}{ }_{1} F_{2}\left(\alpha+1 ; \alpha+\frac{3}{2}, 2 \alpha+1 ; \frac{x^{2}}{4}\right)\right]
\end{aligned}
$$

and

$$
\begin{aligned}
& { }_{1} F_{1}(\alpha ; 2 \alpha ; x) \cdot{ }_{1} F_{1}(\alpha ; 2 \alpha-1 ; x) \\
& =e^{x}\left[{ }_{1} F_{2}\left(\alpha ; \alpha+\frac{1}{2}, 2 \alpha-1 ; \frac{x^{2}}{4}\right)\right. \\
& \left.+\frac{x}{2(2 \alpha-1)}{ }_{1} F_{2}\left(\alpha ; \alpha+\frac{1}{2}, 2 \alpha+1 ; \frac{x^{2}}{4}\right)\right] .
\end{aligned}
$$

Later on, by using the same method employed by Rathie, Rathie and Choi [9] obtained three more results contiguous to (1.1):

$$
\begin{aligned}
& { }_{1} F_{1}(\alpha ; 2 \alpha+1 ; x) \cdot{ }_{1} F_{1}(\alpha ; 2 \alpha-1 ; x) \\
& =e^{x}\left[{ }_{1} F_{2}\left(\alpha ; \alpha+\frac{1}{2}, 2 \alpha-1 ; \frac{x^{2}}{4}\right)\right. \\
& +\frac{x}{2(2 \alpha-1)}{ }_{1} F_{2}\left(\alpha ; \alpha+\frac{1}{2}, 2 \alpha ; \frac{x^{2}}{4}\right) \\
& -\frac{x}{2(2 \alpha+1)}{ }_{2} F_{3}\left(\alpha, \alpha+\frac{1}{2} ; 2 \alpha, \alpha-\frac{1}{2}, \alpha+\frac{3}{2} ; \frac{x^{2}}{4}\right) \\
& \left.+\frac{x^{2}}{4\left(4 \alpha^{2}-1\right)}{ }_{1} F_{2}\left(\alpha+1 ; \alpha+\frac{3}{2}, 2 \alpha+1 ; \frac{x^{2}}{4}\right)\right] ; \\
& { }_{1} F_{1}(\alpha ; 2 \alpha+1 ; x) \cdot{ }_{1} F_{1}(\alpha ; 2 \alpha+1 ; x) \\
& =e^{x}\left[{ }_{1} F_{2}\left(\alpha ; \alpha+\frac{1}{2}, 2 \alpha ; \frac{x^{2}}{4}\right)\right. \\
& -\frac{x}{2 \alpha+1}{ }_{1} F_{2}\left(\alpha+1 ; \alpha+\frac{3}{2}, 2 \alpha+1 ; \frac{x^{2}}{4}\right) \\
& \left.+\frac{x^{2}}{4(2 \alpha+1)^{2}}{ }_{1} F_{2}\left(\alpha+1 ; \alpha+\frac{3}{2}, 2 \alpha+2 ; \frac{x^{2}}{4}\right)\right] ;
\end{aligned}
$$




$$
\begin{aligned}
& { }_{1} F_{1}(\alpha ; 2 \alpha-1 ; x) \cdot{ }_{1} F_{1}(\alpha ; 2 \alpha-1 ; x) \\
& =e^{x}\left[{ }_{1} F_{2}\left(\alpha-1 ; \alpha-\frac{1}{2}, 2 \alpha-2 ; \frac{x^{2}}{4}\right)\right. \\
& +\frac{x}{2 \alpha-1}{ }_{1} F_{2}\left(\alpha ; \alpha+\frac{1}{2}, 2 \alpha-1 ; \frac{x^{2}}{4}\right) \\
& \left.+\frac{x^{2}}{4(2 \alpha-1)^{2}}{ }_{1} F_{2}\left(\alpha ; \alpha+\frac{1}{2}, 2 \alpha ; \frac{x^{2}}{4}\right)\right] .
\end{aligned}
$$

In this paper the results (1.3) to (1.7), obtained by Rathie [8] and Rathie and Choi [9], will be derived with the help of summation formulas contiguous to classical Waton's and Dixon's theorems obtained by Lavoie et al. [4, 5]. It is also shown that three results $(1.5)$ to (1.7) can be rewritten in a compact form.

\section{Results required}

The following known summation formulas due to Lavoie, Grondin and Rathie [4] contiguous to the classical Watson's theorem, and Lavoie, Grondin, Rathie and Arora [5] contiguous to the classical Dixon's theorem and Kummer's first theorem will be required in our present investigations.

Summation formulas contiguous to the classical Watson's theorem:

$$
\begin{aligned}
& { }_{3} F_{2}\left(\begin{array}{c|c}
a, b, & c \\
\frac{1}{2}(a+b+1), & 2 c-1
\end{array}\right) \\
& =\frac{2^{a+b-2} \Gamma\left(\frac{1}{2} a+\frac{1}{2} b+\frac{1}{2}\right) \Gamma\left(c-\frac{1}{2}\right) \Gamma\left(c-\frac{1}{2} a-\frac{1}{2} b-\frac{1}{2}\right)}{\Gamma\left(\frac{1}{2}\right) \Gamma(a) \Gamma(b)} \\
& \cdot\left[\frac{\Gamma\left(\frac{1}{2} a\right) \Gamma\left(\frac{1}{2} b\right)}{\Gamma\left(c-\frac{1}{2} a-\frac{1}{2}\right) \Gamma\left(c-\frac{1}{2} b-\frac{1}{2}\right)}+\frac{\Gamma\left(\frac{1}{2} a+\frac{1}{2}\right) \Gamma\left(\frac{1}{2} b+\frac{1}{2}\right)}{\Gamma\left(c-\frac{1}{2} a\right) \Gamma\left(c-\frac{1}{2} b\right)}\right] \\
& { }_{3} F_{2}\left(\begin{array}{c}
a, \quad(2 c-a-b)>1) ; \\
\frac{1}{2}(a+b+1), \quad 2 c+1
\end{array}\right) \\
& =\frac{2^{a+b-2} \Gamma\left(\frac{1}{2} a+\frac{1}{2} b+\frac{1}{2}\right) \Gamma\left(c+\frac{1}{2}\right) \Gamma\left(c-\frac{1}{2} a-\frac{1}{2} b+\frac{1}{2}\right)}{\Gamma\left(\frac{1}{2}\right) \Gamma(a) \Gamma(b)}
\end{aligned}
$$




$$
\begin{aligned}
& {\left[\frac{\Gamma\left(\frac{1}{2} a\right) \Gamma\left(\frac{1}{2} b\right)}{\Gamma\left(c-\frac{1}{2} a+\frac{1}{2}\right) \Gamma\left(c-\frac{1}{2} b+\frac{1}{2}\right)}-\frac{\Gamma\left(\frac{1}{2} a+\frac{1}{2}\right) \Gamma\left(\frac{1}{2} b+\frac{1}{2}\right)}{\Gamma\left(c-\frac{1}{2} a+1\right) \Gamma\left(c-\frac{1}{2} b+1\right)}\right]} \\
& (\Re(2 c-a-b)>-1) ;
\end{aligned}
$$

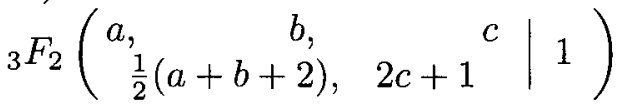

$$
\begin{aligned}
& =\frac{2^{a+b-2} \Gamma\left(\frac{1}{2} a+\frac{1}{2} b+1\right) \Gamma\left(c+\frac{1}{2}\right) \Gamma\left(c-\frac{1}{2} a-\frac{1}{2} b\right)}{(a-b) \Gamma\left(\frac{1}{2}\right) \Gamma(a) \Gamma(b)} \\
& \cdot\left[\frac{(2 c-a+b) \Gamma\left(\frac{1}{2} a+\frac{1}{2}\right) \Gamma\left(\frac{1}{2} b\right)}{\Gamma\left(c-\frac{1}{2} a+1\right) \Gamma\left(c-\frac{1}{2} b+\frac{1}{2}\right)}-\frac{(2 c+a-b) \Gamma\left(\frac{1}{2} a\right) \Gamma\left(\frac{1}{2} b+\frac{1}{2}\right)}{\Gamma\left(c-\frac{1}{2} a+\frac{1}{2}\right) \Gamma\left(c-\frac{1}{2} b+1\right)}\right] \\
& (\Re(2 c-a-b)>0) \text {; }
\end{aligned}
$$

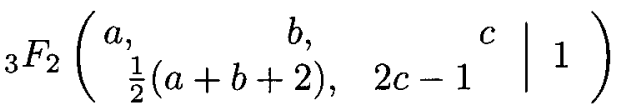

$$
\begin{aligned}
& =\frac{2^{a+b-1} \Gamma\left(\frac{1}{2} a+\frac{1}{2} b+1\right) \Gamma\left(c-\frac{1}{2}\right) \Gamma\left(c-\frac{1}{2} a-\frac{1}{2} b\right)}{(a-b) \Gamma\left(\frac{1}{2}\right) \Gamma(a) \Gamma(b)} \\
& \cdot\left[\frac{\Gamma\left(\frac{1}{2} a+\frac{1}{2}\right) \Gamma\left(\frac{1}{2} b\right)}{\Gamma\left(c-\frac{1}{2} a\right) \Gamma\left(c-\frac{1}{2} b-\frac{1}{2}\right)}-\frac{\Gamma\left(\frac{1}{2} a\right) \Gamma\left(\frac{1}{2} b+\frac{1}{2}\right)}{\Gamma\left(c-\frac{1}{2} a-\frac{1}{2}\right) \Gamma\left(c-\frac{1}{2} b\right)}\right] \\
& (\Re(2 c-a-b)>0) \text {; }
\end{aligned}
$$

$$
\begin{aligned}
& { }_{3} F_{2}\left(\begin{array}{cc|c}
a, & b, & c \\
\frac{1}{2}(a+b), & 2 c+1 & 1
\end{array}\right) \\
& =\frac{2^{a+b-2} \Gamma\left(\frac{1}{2} a+\frac{1}{2} b\right) \Gamma\left(c+\frac{1}{2}\right) \Gamma\left(c-\frac{1}{2} a-\frac{1}{2} b+1\right)}{\Gamma\left(\frac{1}{2}\right) \Gamma(a) \Gamma(b)} \\
& \cdot\left[\frac{\Gamma\left(\frac{1}{2} a+\frac{1}{2}\right) \Gamma\left(\frac{1}{2} b\right)}{\Gamma\left(c-\frac{1}{2} a+1\right) \Gamma\left(c-\frac{1}{2} b+\frac{1}{2}\right)}+\frac{\Gamma\left(\frac{1}{2} a\right) \Gamma\left(\frac{1}{2} b+\frac{1}{2}\right)}{\Gamma\left(c-\frac{1}{2} a+\frac{1}{2}\right) \Gamma\left(c-\frac{1}{2} b+1\right)}\right] \\
& (\Re(2 c-a-b)>-2) .
\end{aligned}
$$

Summation formulas contiguous to the classical Dixon's theorem:

$$
\begin{aligned}
& { }_{3} F_{2}\left(\begin{array}{cc|c}
a, \quad b, & c & 1 \\
2+a-b, & 2+a-c & 1
\end{array}\right) \\
& =\frac{2^{1-2 c} \Gamma(2+a-b) \Gamma(2+a-c)}{(b-1)(c-1) \Gamma(a-2 c+2) \Gamma(a-b-c+2)}
\end{aligned}
$$




$$
\begin{gathered}
\cdot\left[\frac{\Gamma\left(\frac{1}{2} a-c+\frac{3}{2}\right) \Gamma\left(\frac{1}{2} a-b-c+2\right)}{\Gamma\left(\frac{1}{2} a+\frac{1}{2}\right) \Gamma\left(\frac{1}{2} a-b+1\right)}\right. \\
\left.-\frac{\Gamma\left(\frac{1}{2} a-c+1\right) \Gamma\left(\frac{1}{2} a-b-c+\frac{5}{2}\right)}{\Gamma\left(\frac{1}{2} a\right) \Gamma\left(\frac{1}{2} a-b+\frac{3}{2}\right)}\right] \\
(\Re(a-2 b-2 c)>-4) ;
\end{gathered}
$$

$$
\begin{aligned}
& { }_{3} F_{2}\left(\begin{array}{cc|c}
a, & b, & 1 \\
a-b, & a-c & 1
\end{array}\right) \\
& =\frac{2^{-2 c-1} \Gamma(a-b) \Gamma(a-c)}{\Gamma(a-2 c) \Gamma(a-b-c)} \\
& \cdot\left[\frac{\Gamma\left(\frac{1}{2} a-c+\frac{1}{2}\right) \Gamma\left(\frac{1}{2} a-b-c\right)}{\Gamma\left(\frac{1}{2} a+\frac{1}{2}\right) \Gamma\left(\frac{1}{2} a-b\right)}+\frac{\Gamma\left(\frac{1}{2} a-c\right) \Gamma\left(\frac{1}{2} a-b-c+\frac{1}{2}\right)}{\Gamma\left(\frac{1}{2} a\right) \Gamma\left(\frac{1}{2} a-b+\frac{1}{2}\right)}\right] \\
& (\Re(a-2 b-2 c)>0) ; \\
& { }_{3} F_{2}\left(\begin{array}{c|c}
a, \\
a-b,
\end{array}\right) \\
& =\frac{2^{-2 c-2} \Gamma(a-b) \Gamma(a-c-1)}{\Gamma(a-2 c-1) \Gamma(a-b-c)}
\end{aligned}
$$

$$
\begin{aligned}
& \cdot\left[\frac{(a-c-1) \Gamma\left(\frac{1}{2} a-c-\frac{1}{2}\right) \Gamma\left(\frac{1}{2} a-b-c\right)}{\Gamma\left(\frac{1}{2} a+\frac{1}{2}\right) \Gamma\left(\frac{1}{2} a-b\right)}\right. \\
& \left.\quad+\frac{(a-2 b-c-1) \Gamma\left(\frac{1}{2} a-c\right) \Gamma\left(\frac{1}{2} a-b-c-\frac{1}{2}\right)}{\Gamma\left(\frac{1}{2} a\right) \Gamma\left(\frac{1}{2} a-b+\frac{1}{2}\right)}\right] \\
& \quad(\Re(a-2 b-2 c)>1) ;
\end{aligned}
$$

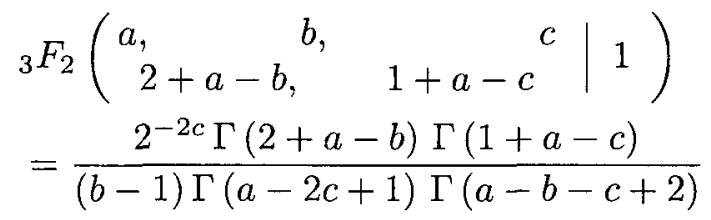

$$
\begin{gathered}
\cdot\left[\frac{\Gamma\left(\frac{1}{2} a-c+1\right) \Gamma\left(\frac{1}{2} a-b-c+\frac{3}{2}\right)}{\Gamma\left(\frac{1}{2} a\right) \Gamma\left(\frac{1}{2} a-b+\frac{3}{2}\right)}\right. \\
\left.-\frac{\Gamma\left(\frac{1}{2} a-c+\frac{1}{2}\right) \Gamma\left(\frac{1}{2} a-b-c+2\right)}{\Gamma\left(\frac{1}{2} a+\frac{1}{2}\right) \Gamma\left(\frac{1}{2} a-b+1\right)}\right] \\
(\Re(a-2 b-2 c)>-3) ;
\end{gathered}
$$




$$
\begin{gathered}
{ }_{3} F_{2}\left(\begin{array}{cc|c}
a, & b, & c \\
a-b, & 1+a-c & 1
\end{array}\right) \\
=\frac{2^{-2 c} \Gamma(a-b) \Gamma(1+a-c)}{\Gamma(a-2 c+1) \Gamma(a-b-c+1)} \\
\cdot\left[\frac{\Gamma\left(\frac{1}{2} a-c+\frac{1}{2}\right) \Gamma\left(\frac{1}{2} a-b-c+1\right)}{\Gamma\left(\frac{1}{2} a+\frac{1}{2}\right) \Gamma\left(\frac{1}{2} a-b\right)}\right. \\
\left.+\frac{\Gamma\left(\frac{1}{2} a-c+1\right) \Gamma\left(\frac{1}{2} a-b-c+\frac{1}{2}\right)}{\Gamma\left(\frac{1}{2} a\right) \Gamma\left(\frac{1}{2} a-b+\frac{1}{2}\right)}\right] \\
(\Re(a-2 b-2 c)>-1) .
\end{gathered}
$$

Kummer's first theorem [3]:

$$
e^{-x}{ }_{1} F_{1}(\rho ; \sigma ; x)={ }_{1} F_{1}(\sigma-\rho ; \sigma ;-x)
$$

\section{Some useful contiguous relations}

Before writing the results (1.5) to (1.7) in compact forms, first we shall derive the following interesting and useful contiguous relations:

$$
\begin{array}{r}
{ }_{1} F_{2}\left(\alpha ; \alpha+\frac{1}{2}, 2 \alpha ; \frac{x^{2}}{4}\right)={ }_{1} F_{2}\left(\alpha ; \alpha+\frac{1}{2}, 2 \alpha-1 ; \frac{x^{2}}{4}\right) \\
-\frac{x^{2}}{4\left(4 \alpha^{2}-1\right)}{ }_{1} F_{2}\left(\alpha+1 ; \alpha+\frac{3}{2}, 2 \alpha+1 ; \frac{x^{2}}{4}\right) \\
{ }_{1} F_{2}\left(\alpha ; \alpha+\frac{3}{2}, 2 \alpha ; \frac{x^{2}}{4}\right)=\frac{2 \alpha+1}{2}{ }_{1} F_{2}\left(\alpha ; \alpha+\frac{1}{2}, 2 \alpha ; \frac{x^{2}}{4}\right) \\
-\frac{2 \alpha-1}{2}{ }_{2} F_{3}\left(\alpha, \alpha+\frac{1}{2} ; \alpha-\frac{1}{2}, \alpha+\frac{3}{2}, 2 \alpha ; \frac{x^{2}}{4}\right) \\
{ }_{1} F_{2}\left(\alpha+1 ; \alpha+\frac{1}{2}, 2 \alpha+1 ; \frac{x^{2}}{4}\right)={ }_{1} F_{2}\left(\alpha ; \alpha+\frac{1}{2}, 2 \alpha ; \frac{x^{2}}{4}\right) \\
+\frac{x^{2}}{4(2 \alpha+1)^{2}}{ }_{1} F_{2}\left(\alpha+1 ; \alpha+\frac{3}{2}, 2 \alpha+2 ; \frac{x^{2}}{4}\right)
\end{array}
$$




$$
\begin{gathered}
{ }_{1} F_{2}\left(\alpha ; \alpha-\frac{1}{2}, 2 \alpha-1 ; \frac{x^{2}}{4}\right)={ }_{1} F_{2}\left(\alpha-1 ; \alpha-\frac{1}{2}, 2 \alpha-2 ; \frac{x^{2}}{4}\right) \\
+\frac{x^{2}}{4(2 \alpha-1)^{2}}{ }_{1} F_{2}\left(\alpha ; \alpha+\frac{1}{2}, 2 \alpha ; \frac{x^{2}}{4}\right) .
\end{gathered}
$$

To prove (3.1), denote the right-hand side of (3.1), we have

$$
\begin{aligned}
I & =\sum_{n=0}^{\infty} \frac{(\alpha)_{n} x^{2 n}}{(2 \alpha-1)_{n}\left(\alpha+\frac{1}{2}\right)_{n} 2^{2 n} n !} \\
& -\frac{x^{2}}{4\left(4 \alpha^{2}-1\right)} \sum_{n=0}^{\infty} \frac{(\alpha+1)_{n} x^{2 n}}{(2 \alpha+1)_{n}\left(\alpha+\frac{3}{2}\right)_{n} 2^{2 n} n !},
\end{aligned}
$$

which, upon replacing $n$ by $n-1$ in the second summation, leads to

$$
\begin{aligned}
I & =1+\sum_{n=1}^{\infty} \frac{(\alpha)_{n} x^{2 n}}{(2 \alpha-1)_{n}\left(\alpha+\frac{1}{2}\right)_{n} 2^{2 n} n !} \\
& -\sum_{n=1}^{\infty} \frac{\left(\alpha+\frac{1}{2}\right) 2 \alpha(2 \alpha-1)(\alpha)_{n} x^{2 n}}{\left(4 \alpha^{2}-1\right) \alpha(2 \alpha+n-1)(2 \alpha-1)_{n}\left(\alpha+\frac{1}{2}\right)_{n} 2^{2 n}(n-1) !} \\
& =1+\sum_{n=1}^{\infty} \frac{(\alpha)_{n} x^{2 n}}{(2 \alpha-1)_{n}\left(\alpha+\frac{1}{2}\right)_{n} 2^{2 n} n !}\left(1-\frac{n}{2 \alpha+n-1}\right) \\
& =1+\sum_{n=1}^{\infty} \frac{(\alpha)_{n} x^{2 n}}{(2 \alpha)_{n}\left(\alpha+\frac{1}{2}\right)_{n} 2^{2 n} n !} \\
& ={ }_{1} F_{2}\left(\alpha ; \alpha+\frac{1}{2}, 2 \alpha ; \frac{x^{2}}{4}\right),
\end{aligned}
$$

which is the left-hand side of (3.1).

Similarly we can prove (3.2) to (3.4).

\section{Proofs of (1.3) to (1.7)}

In order to prove (1.3), it is easy to see that, in view of (2.11), (1.3) can be written as

$$
\begin{aligned}
& { }_{1} F_{1}(\alpha ; 2 \alpha ; x) \cdot{ }_{1} F_{1}(\alpha+1 ; 2 \alpha+1 ;-x) \\
& \quad={ }_{1} F_{2}\left(\alpha ; 2 \alpha, \alpha+\frac{1}{2} ; \frac{x^{2}}{4}\right) \\
& \quad-\frac{x}{2(2 \alpha+1)}{ }_{1} F_{2}\left(\alpha+1 ; 2 \alpha+1, \alpha+\frac{3}{2} ; \frac{x^{2}}{4}\right) .
\end{aligned}
$$


In order to prove (4.1), we proceed as follows:

$$
\begin{aligned}
& { }_{1} F_{1}(\alpha ; 2 \alpha ; x) \cdot{ }_{1} F_{1}(\alpha+1 ; 2 \alpha+1 ;-x) \\
& =\sum_{n=0}^{\infty} \sum_{m=0}^{\infty} \frac{(\alpha)_{n} x^{n}}{(2 \alpha)_{n} n !} \frac{(\alpha+1)_{m}(-1)^{m} x^{m}}{(2 \alpha+1)_{m} m !} \\
& =\sum_{n=0}^{\infty} \sum_{m=0}^{n} \frac{(\alpha)_{n-m}(\alpha+1)_{m}(-1)^{m} x^{n}}{(2 \alpha)_{n-m}(n-m) !(2 \alpha+1)_{m} m !} .
\end{aligned}
$$

Now, if we use the well-known identities:

$$
(\alpha)_{n-m}=\frac{(-1)^{m}(\alpha)_{n}}{(1-\alpha-n)_{m}} \quad \text { and } \quad(n-m) !=\frac{(-1)^{m} n !}{(-n)_{m}},
$$

after a little simplification, we have

$$
\begin{aligned}
& { }_{1} F_{1}(\alpha ; 2 \alpha ; x) \cdot{ }_{1} F_{1}(\alpha+1 ; 2 \alpha+1 ;-x) \\
& =\sum_{n=0}^{\infty} \frac{(\alpha)_{n} x^{n}}{(2 \alpha)_{n} n !}{ }_{3} F_{2}\left(\begin{array}{c|c}
-n, & 1-2 \alpha-n, \\
1-\alpha-n, & \alpha+1
\end{array}\right) \\
& =\sum_{n=0}^{\infty} \frac{(\alpha)_{2 n} x^{2 n}}{(2 \alpha)_{2 n}(2 n) !}{ }_{3} F_{2}\left(\begin{array}{cc|c}
-2 n, & 1-2 \alpha-2 n, & \alpha+1 \\
1-\alpha-2 n, & 2 \alpha+1 & 1
\end{array}\right) \\
& +\sum_{n=0}^{\infty} \frac{(\alpha)_{2 n+1} x^{2 n+1}}{(2 \alpha)_{2 n+1}(2 n+1) !} \\
& { }_{3} F_{2}\left(\begin{array}{rr|r}
-2 n-1, & -2 \alpha-2 n, & \alpha+1 \\
-\alpha-2 n, & 2 \alpha+1 & 1
\end{array}\right) .
\end{aligned}
$$

In (4.2), for the first ${ }_{3} F_{2}$, by taking $a=-2 n, b=1-2 \alpha-2 n$ and $c=\alpha+1$ and for the second ${ }_{3} F_{2}$, by putting $a=-2 n-1, b=-2 \alpha-2 n$ and $c=\alpha+1$ in the contiguous Watson's theorem (2.1), after a little simplification, we get

$$
\begin{aligned}
& { }_{1} F_{1}(\alpha ; 2 \alpha ; x) \cdot{ }_{1} F_{1}(\alpha+1 ; 2 \alpha+1 ;-x) \\
& =\sum_{n=0}^{\infty} \frac{(\alpha)_{n} x^{2 n}}{(2 \alpha)_{n}\left(\alpha+\frac{1}{2}\right)_{n} 2^{2 n} n !} \\
& -\frac{1}{2(2 \alpha+1)} \sum_{n=0}^{\infty} \frac{(\alpha+1)_{n} x^{2 n+1}}{(2 \alpha+1)_{n}\left(\alpha+\frac{3}{2}\right)_{n} 2^{2 n} n !}
\end{aligned}
$$


which is seen to be the right-hand side of (4.1).

It is noted that in (4.2), for the first ${ }_{3} F_{2}$, by taking $a=-2 n, b=\alpha+1$ and $c=1-2 \alpha-2 n$ and for the second ${ }_{3} F_{2}$, by putting $a=-2 n-1$, $b=\alpha+1$ and $c=-2 \alpha-2 n$ in the contiguous Dixon's theorem (2.6), after a little simplification, we arrive at the same expression as in (4.3).

In the exactly same manner, (1.4) can also be derived.

In order to prove (1.5), with the help of (3.1) and (3.2), (1.5) can be written in the following compact form:

$$
\begin{aligned}
& { }_{1} F_{1}(\alpha ; 2 \alpha+1 ; x) \cdot{ }_{1} F_{1}(\alpha ; 2 \alpha-1 ; x) \\
& \quad=e^{x}\left[{ }_{1} F_{2}\left(\alpha ; \alpha+\frac{1}{2}, 2 \alpha ; \frac{x^{2}}{4}\right)\right. \\
& \left.\quad+\frac{x}{4 \alpha^{2}-1}{ }_{1} F_{2}\left(\alpha ; \alpha+\frac{3}{2}, 2 \alpha ; \frac{x^{2}}{4}\right)\right],
\end{aligned}
$$

which now can be proved by using the same technique as in deriving (1.3).

Similarly (1.6) and (1.7) can also be written in the following compact forms by using the contiguous relations (3.3) and (3.4) as follows:

$$
\begin{aligned}
& { }_{1} F_{1}(\alpha ; 2 \alpha+1 ; x) \cdot{ }_{1} F_{1}(\alpha ; 2 \alpha+1 ; x) \\
& =e^{x}\left[{ }_{1} F_{2}\left(\alpha+1 ; \alpha+\frac{1}{2}, 2 \alpha+1 ; \frac{x^{2}}{4}\right)\right. \\
& \left.\quad-\frac{x}{2 \alpha+1}{ }_{1} F_{2}\left(\alpha+1 ; \alpha+\frac{3}{2}, 2 \alpha+1 ; \frac{x^{2}}{4}\right)\right]
\end{aligned}
$$

and

$$
\begin{aligned}
& { }_{1} F_{1}(\alpha ; 2 \alpha-1 ; x) \cdot{ }_{1} F_{1}(\alpha ; 2 \alpha-1 ; x) \\
& =e^{x}\left[{ }_{1} F_{2}\left(\alpha ; \alpha-\frac{1}{2}, 2 \alpha-1 ; \frac{x^{2}}{4}\right)\right. \\
& \left.\quad+\frac{x}{2 \alpha-1}{ }_{1} F_{2}\left(\alpha ; \alpha+\frac{1}{2}, 2 \alpha-1 ; \frac{x^{2}}{4}\right)\right] .
\end{aligned}
$$

It is remaked that, for other interesting generalizations of the Preece's identity (1.1), see Rathie and Choi [10], and Rathie and Sharma [11]. 


\section{References}

[1] W. N. Bailey, Products of generalized hypergeometric series, Proc. London Math. Soc.(2) 28 (1928), 242-254.

[2] Y. S. Kim and A. K. Rathie, A generalization of Preece's identity, Commun. Korean Math. Soc. 14 (1999), 217-222.

[3] E. E. Kummer, Über die hypergeometrische Reihe $F(a ; b ; x)$, J. Reine Angew. Math. 15 (1836), 39-83.

[4] J. L. Lavoie, F. Grondin, and A. K. Rathie, Generalizations of Watson's theorem on the sum of $a_{3} F_{2}$, Indian J. Math. 34 (1992), 23-32.

[5] J. L. Lavoie, F. Grondin, A. K. Rathie, and K. Arora, Generalizations of Dixon's theorem on the sum of $a_{3} F_{2}$, Math. Comp. 49 (1987), 269-274.

[6] C. T. Preece, The product of two generalized hypergeometric functions, Proc. London Math. Soc.(2) 24 (1924), 370-380.

[7] E. D. Rainville, Special Functions, The Macmillan Company, New York, 1960.

[8] A. K. Rathie, A short proof of Preece's identity and other contiguous results, Rev. Mat. Estatist.(Brazil) 15 (1997), 207-210.

[9] A. K. Rathie and J. Choi, A note on an identity due to Preece, Far East J. Math. Sci. 6 (1998), 205-209.

[10] _ results, Bull. Korean Math. Soc. 35 (1998), 339-344.

[11] A. K. Rathie and S. Sharma, On a generalization of Preece's identity, Acta Cienc. Indica Math. 105 (1999), 167-168.

\section{Junesang Choi}

Department of Mathematics

College of Natural Sciences

Dongguk University

Kyongju 780-714, Korea

E-mail: junesang@mail.dongguk.ac.kr

Arjun K. Rathie and Bharti Bhojak

Department of Mathematics

Dungar College

Bikaner 334001, Rajasthan State, India

E-mail: akrathie@rediffmail.com 